\title{
MÉTODOS DIAGNÓSTICOS DE LA DISEMINACIÓN PROSTÁTICA.
}

\author{
José Antonio Lorente Garín y Antoni Gelabert Mas.
}

Servicio y Cátedra de Urología. Hospital del Mar. Universidad Autónoma de Barcelona. Barcelona. España.

\begin{abstract}
Resumen.- El cáncer de próstata (CP) metastatiza a distancia preferentemente en los ganglios linfáticos regionales y en el hueso. La incidencia de la diseminación se ha visto reducida en los últimos años debido principalmente al amplio uso del antígeno prostático específico (PSA). Por este motivo, la indicación de la realización de las exploraciones complementarias ha ido evolucionando en un intento de mejorar su rentabilidad. Algunas de estas técnicas se encuentran actualmente en evaluación y pueden contribuir en un próximo futuro a cambiar la práctica clínica del estudio de extensión. En la evaluación de la diseminación ganglionar, el TC o la RM son las técnicas por imagen estándar mientras que en la evaluación ósea la GO continúa siendo el rastreo de rutina.
\end{abstract}

Palabras clave: Cáncer de próstata. Diseminación. Métodos diagnósticos.

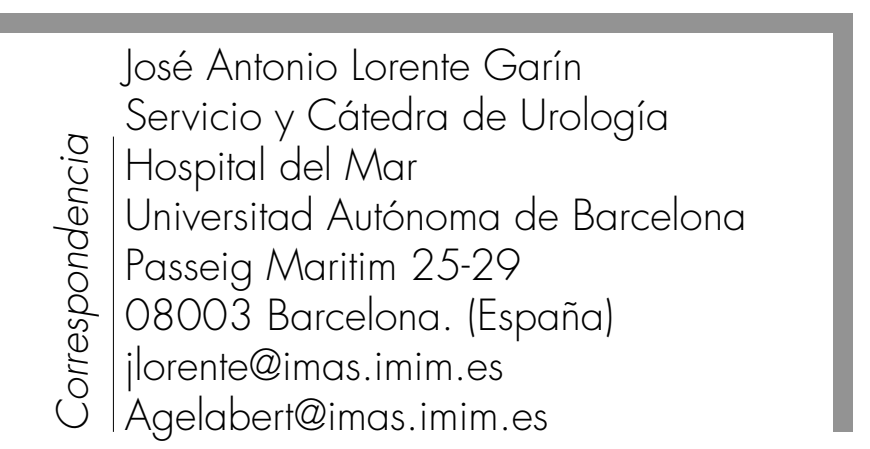

\begin{abstract}
Summary.- Prostate cancer preferentially metastasizes to regional lymph nodes and bone. The incidence of dissemination has been reduced over the last years mainly due to the compost use of PSA. For this reason, the indication of complementary diagnostic tests has evolved with the aim of improving the diagnostic yield. Some of these techniques are currently under evaluation and may contribute in the close future to change the study of dissemination in the clinical practice. CT scan or MRl are the standard imaging studies for lymph node dissemination, whereas bone scan continues to be the routine test for bone dissemination.
\end{abstract}

Keywords: Prostate cancer. Disemination. Diagnostic methods.

\section{INTRODUCCIÓN}

El cáncer de próstata (CP) metastatiza a distancia preferentemente en los ganglios linfáticos regionales y en el hueso, presentándose en más del $95 \%$ de los pacientes en la autopsia. Las metástasis óseas (m.o.) en la pelvis y columna lumbar se producen con una frecuencia mucho mayor de la esperada si la vascularización de estas localizaciones fuera la única determinante de la distribución de los focos metastáticos. Esta bien documentada tanto la ruta de diseminación hematógena por los plexos paravertebrales como la cascada de pasos secuenciales que las células tumorales deben superar hasta anidar en el órgano diana. La incidencia de la diseminación ganglionar y ósea en el CP se ha visto reducida en los últimos años debido principalmente al amplio uso del antígeno prostático específico (PSA). Esta práctica generalizada ha demostrado la migración 
hacia estadios más precoces del $\mathrm{CP}$ en el momento del diagnóstico. Por este motivo, la indicación de la realización de las exploraciones complementarias ha ido evolucionando en un intento de mejorar su rentabilidad. En la actualidad, disponemos de técnicas más sensibles y específicas que permiten realizar un mejor estadiaje clínico. En esta revisión se repasan las técnicas de diagnóstico por la imagen para el estudio de la diseminación en el CP.

\section{DIAGNÓSTICO DE LA DISEMINACIÓN GAN- GLIONAR}

\section{Tomografía computerizada (TC)}

La indicación principal de la TC abdominal o pélvica en el CP es la detección de la enfemedad metastásica ganglionar. El papel de la TC en el estadiaje del CP es sin embargo controvertido, debido al reducido porcentaje de estudios positivos $(<4 \%)$. En pacientes asintomáticos con PSA menor de $20 \mathrm{ng} /$ $\mathrm{ml}$, estadios clínicamente localizados o CP de grado moderado no se necesita un estudio con la TC por la baja probabilidad de obtener resultados positivos $(<1 \%)$ (1). La técnica adecuada de TC requiere la administración de contraste endovenoso para facilitar la diferenciación entre los vasos sanguíneos y los nódulos linfáticos, (Figura 1). La definición de adenomagalia por metástasis se basa únicamente en el tamaño, considerándose anormales los mayores de 1 $\mathrm{cm}$ de diámetro en el eje corto (2). La TC no permite definir la arquitectura interna. Los falsos negativos se producen por la metástasis en los ganglios de apariencia normal mientras que los falsos positivos son debidos a adenomagalias generalmente inflamatorias. La sensibilidad varía entre el $30 \%$ y $78 \%$, la especificidad entre el $77 \%$ y $97 \%$ y la eficacia entre el $70 \%$ y $94 \%$ (3). Debido a que un falso positivo puede impedir la realización de un tratamiento potencialmente curativo, toda adenomegalia debería confirmarse mediante estudio patológico sobretodo en los pacientes con cifras bajas de PSA. En algunas series la alta especificidad de la combinación de TC y biopsia con aguja fina se propone como alternativa a la linfadenectomía laparoscópica. En la evaluación inicial, la TC no debe extenderse al estudio del retroperitoneo en ausencia de imágenes ganglionares sospechosas por debajo de la bifurcación ilíaca. Sin embargo, si es aconsejable en la evaluación de la recidiva bioquímica.

\section{Resonancia magnética (RM)}

La diseminación ganglionar también puede ser diagnosticada mediante RM. El criterio para identificar los gánglios como patológicos es atendiendo al tamaño como en la TC. Los nódulos linfáticos tienen baja intensidad de señal en secuencia Tl y mayor en secuencia T2, pudiendo obtener imágenes en otras secuencias para distinguirlos de las estructuras adyacentes. La posibilidad de emplear los contrastes paramagnéticos permiten su uso en los pacientes alérgicos a los contrastes radiológicos. En la literatura se ofrece una eficacia similar a la del TC en esta aplicación aunque se sugiere su empleo al ofrecer mejor información en el estadiaje local (4).

\section{Tomografía por emisión de positrones (PET y PET/TC)}

La PET es una técnica no invasiva que permite la evaluación de los procesos metabólicos en el tejido. El radiotrazador más empleado es la 18FFDG cuya acumulación se correlaciona con la actividad proliferativa. A diferencia de otros cánceres, en el CP tiene poca aplicación debido en parte al bajo consumo de glucosa en la mayoría de los casos. La sensibilidad en la detección del CP es de $60 \%$ a $70 \%$, principalmente $\mathrm{CP}$ de alto grado, estadio avanzado y con altas concentraciones de PSA (5). Se ha descrito recientemente que el CP presenta una captación incrementada de colina que es empleada como sustrato para la síntesis de la fosfatidilcolina de la membrana celular. La PET con 11C-Colina ofrece resultados superiores en la identificación del $C P$ que con 18F-FDG. Otra ventaja añadida es la baja excreción urinaria con mejor definición de las estructuras pélvicas, (Figura 2). Debido al aumento en el metabolismo lipídico producido por la síntesis de la membrana celular, el acetato es otra molécula que puede ser un buen indicador de esta vía metabólica en el tejido tumoral. La limitación de la PET es su reducida resolución espacial pudiendo detectar lesiones a partir de los $5 \mathrm{~mm}$. Los resultados comparativos entre la PET con $11 \mathrm{C}$-Colina y $11 \mathrm{C}$-Acetato ofrecen sensibilidades cercanas al $80 \%$ en el estudio de la enfermedad ganglionar y no dependen de la diferenciación histológica ni del nivel de PSA. La especificidad y la eficacia es del $96 \%$ y $93 \%$, superiores a la del TC o RM (6).

\section{Gammagrafía con ProstaScint}

La gammagrafía con ProstaScint se basa en el radiomarcaje de un anticuerpo monoclonal frente al antígeno prostático específico de membrana con $111 \mathrm{ln}$. Está actualmente autorizado por la FDA para la detección clínica de recidivas y metástasis de $\mathrm{CP}$ en tejidos blandos. Esta técnica se ha sometido a diferentes estudios con una amplio rango de sensibilidad, especificidad y eficacia en el diagnóstico de la diseminación. Los resultados se deben interpretar con cautela y dentro de protocolos clínicos (7). 


\section{DIAGNÓSTICO DE LA DISEMINACIÓN ÓSEA}

\section{Radiología convencional}

La radiología convencional permite la visualización con detalle de las alteraciones morfológicas originadas por las metástasis en el hueso. Generalmente, la formación de hueso nuevo se produce de manera acoplada a la destrucción y el aspecto radiológico final refleja meramente el proceso que predomina. El remodelado no compensado con excesiva neoformación y mínima resorción es la causa de que la mayoría de las m.o. por CP sean radiológicamente osteocondensantes. La radiología convencional es una exploración específica pero poco sensible en el diagnóstico de las lesiones óseas. Esto justifica que el diagnóstico basado en estas técnicas haya subestimado la incidencia real de la afectación metastásica. Se requieren una serie de cambios morfológicos a nivel del contenido del mineral cálcico para hacerse visibles. Una m.o. osteolítica en un cuerpo vertebral tiene que destruir más del $66 \%$ del hueso esponjoso, creciendo más de $1 \mathrm{~cm}$., antes de que pueda ser detectada en una radiografía anteroposterior (8). Mediante proyecciones laterales la visualización es mejor pero todavía se requiere una pérdida del $50 \%$. La respuesta osteoblástica hace la detección radiológica algo más fácil. En la fase precoz de formación ósea no se ha depositado suficiente material, ya que es necesario un mínimo del $30 \%$ de aumento del contenido mineral para que sea visualizada como radiodensa. Por todo ello, durante la fase precoz de la invasión metastásica ósea, una radiografía normal puede acompañarse de otras exploraciones demostrativas de m.o. (9). No es infrecuente la coexistencia

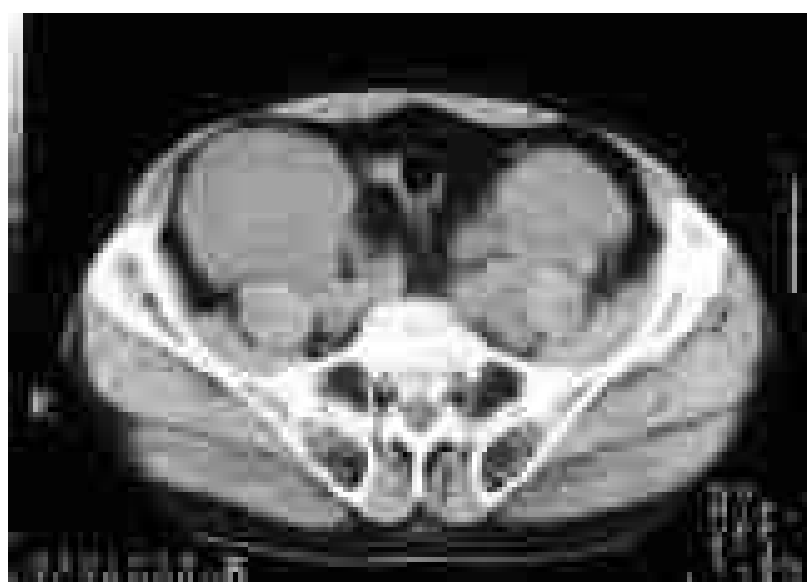

FIGURA 1. TC pélvica que muestra grandes nódulos adenopáticos en un paciente afecto de cáncer de próstata. de focos con diferente comportamiento radiológico, (Figura 3). En un estudio autópsico el diagnóstico radiológico se realizó sólo en el $42 \%$ de las columnas invadidas (10). Si la lesión está más avanzada, la deformación en el contorno del cuerpo vertebral la hacen más fácil de diagnosticar. El examen cuidadoso de los contornos de los cuerpos vertebrales buscando roturas corticales, insuflación o respuestas osteoblásticas en los márgenes ayudan al diagnóstico.

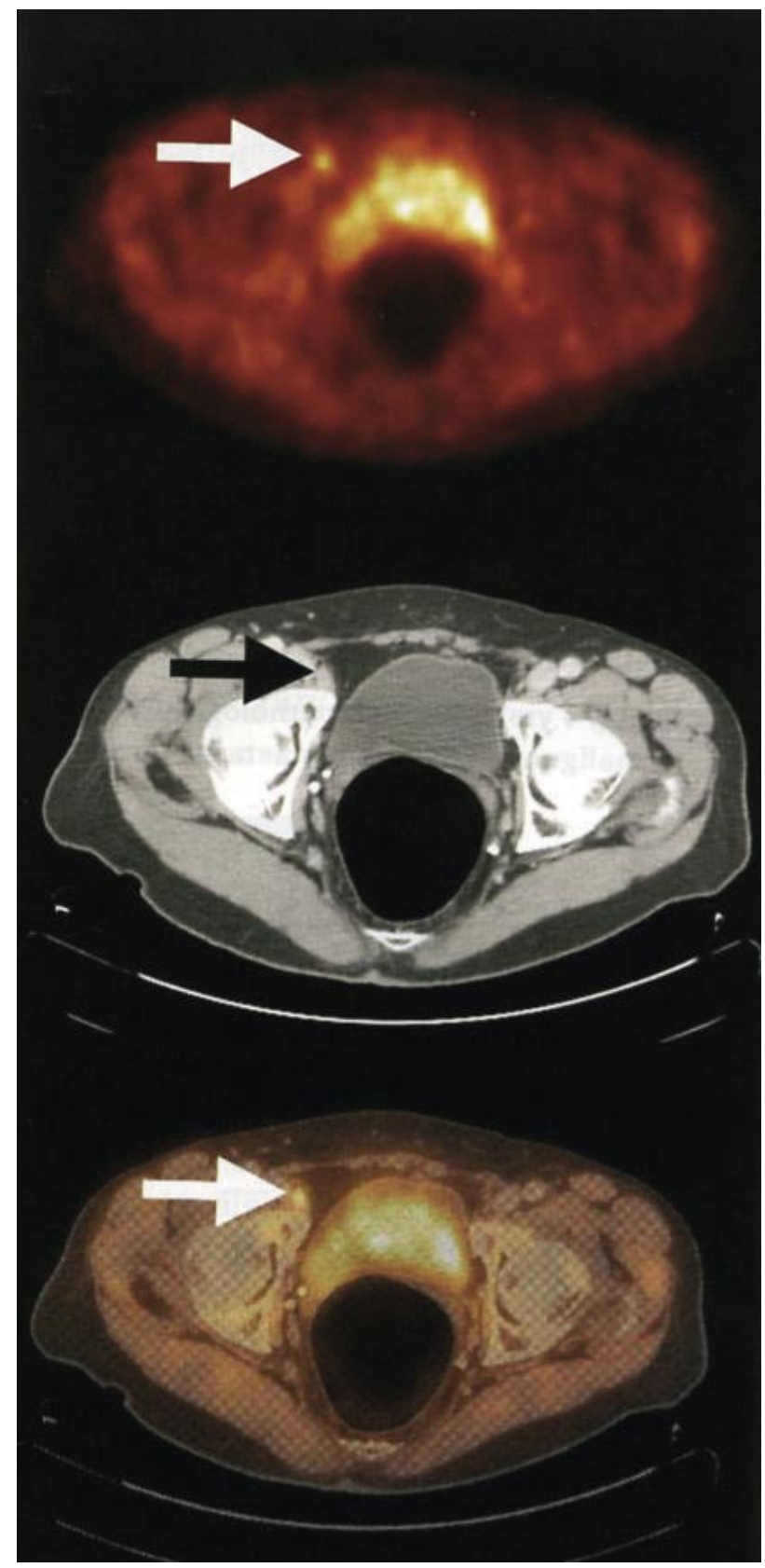

FIGURA 2. PET/TC con 11 C-Colina donde se aprecia una captación en la región ilíaca derecha compatible con un ganglio metastático. (Tomado de DeJong et al.) 
La indicación actual de la radiología convencional se limita a casos concretos susceptibles de evaluación conjunta con otras técnicas como en las m.o. solitarias de difícil interpretación. La radiología permite a su vez detectar la existencia de posibles fracturas en pacientes con dolor óseo y el seguimiento de las lesiones tras la supresión androgénica. El tratamiento puede producir cambios en el remodelado haciendo que lesiones osteolíticas puedan condensar y que algunas blásticas temporalmente aumenten o disminuyan su componente osteoblástico. Se ha comunicado que la evolución radiológica de las lesiones puede tener valor pronóstico (11).

\section{Resonancia magnética}

La RM es una técnica diagnóstica sensible para demostrar focos de m.o. Es capaz de detectar sutiles cambios en la composición de la grasa medular reflejados por alteraciones en la intensidad de la señal. El efecto de las m.o. sobre la señal de la RM está en función de variables como la edad del paciente, la localización de las lesiones, la secuencia del pulso, la naturaleza del foco metastático y el empleo de contraste paramagnéticos (12).

La composición de la médula varía con la edad, produciéndose una progresiva sustitución de la médula ósea roja hematopoyética por tejido graso desde el centro del cuerpo vertebral hacia la periferia. Esta sustitución es cronológicamente ascendente siendo las últimas vértebras reemplazadas la primera lumbar y la última dorsal. Esta situación facilita la interpretación de las alteraciones de la intensidad de la señal. Básicamente, el corto tiempo de relajación

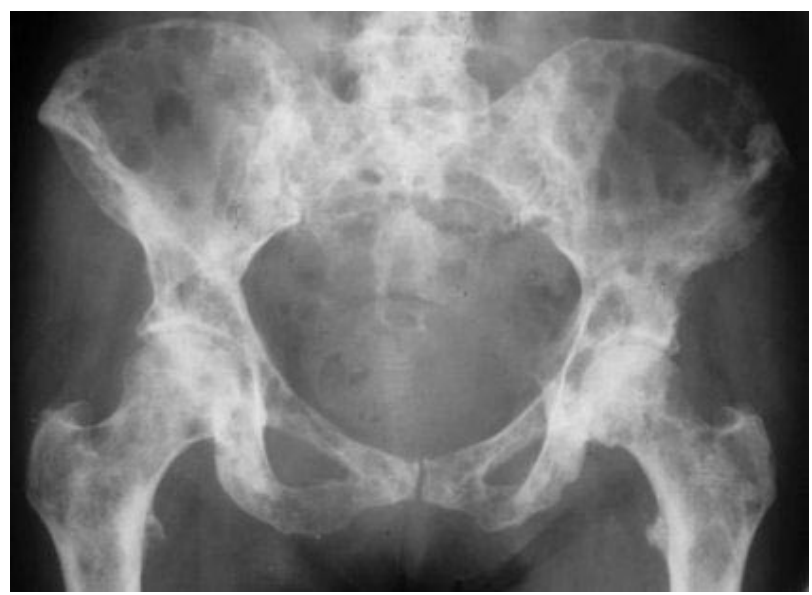

FIGURA 3. Radiografía pélvica en un paciente con múltiples imágenes de metástasis óseas por cáncer de próstata con diferentes patrones radiológicos. en secuencia Tl de la grasa comparada con la del agua permite que la médula ósea del adulto tenga normalmente alta señal. Análogamente, en secuencia T2 sucede algo parecido (13). En principio y en el estudio de esta localización, la cantidad de mineral óseo afecta poco la señal magnética. Sin embargo, al aumentar la masa calcica se produce una menor señal por la menor movilidad de los protones en el hueso compacto. Es manifiesta la utilidad de las secuencias T1 y T2 en casos de m.o. Otras secuencias, como la supresión grasa, pueden aportar información en el diagnóstico diferencial de las fracturas patológicas (14). Los focos metastásicos prostáticos son moderadamente hipervasculares y se encuentran en una situación intermedia en intensidad de la señal final, dependiendo ésta de la relación entre reacción esclerótica y el osteoide no calcificado. El foco metastásico casi siempre se presenta como una señal reducida en $\mathrm{T} 1$ mientras la grasa medular adyacente presenta una alta señal. En secuencia T2 es más variable y depende en mayor medida del tipo de tejido, del contenido en agua y de la presencia de fibrosis, necrosis, hematoma, esclerosis y exudado inflamatorio. La intensidad de la señal puede no ser uniforme en función de la existencia de componente mixto (15). La sensibilidad de la RM para el diagnóstico de las m.o. por CP es elevada variando en las distintas series $(16,17)$. Es semejante a la obtenida con la gammagrafía ósea (GO) e incluso mayor (18). En un estudio comparativo (19) la RM fue informada como confirmativa de afectación metastásica en el $25 \%$ de los casos de GO dudosa o no concluyente. En este mismo estudio la RM contribuyó a detectar más focos de los observados en la gammagrafía en un tercio de los pacientes. La especificidad parece mayor por que permite distinguir más casos de patologías óseas benignas. La afectación de partes blandas es detectada bien mediante RM por lo que su indicación en caso de afectación epidural con compromiso medular es mandataria, (Figura 4). Su limitación viene dada por la imposibilidad de realización de rastreos corporales totales, si bien es la técnica de elección en el estudio de focos gammagráficos de dudosa naturaleza.

\section{Gammagrafía ósea (GO)}

La GO es actualmente la principal exploración para el estudio de la extensión al hueso del CP. Se puede definir como una exploración funcional del tejido óseo, consistente en la obtención de imágenes de todo el esqueleto tras la fijación en el mismo de un radiotrazador con tropismo óseo. La captación del radiotrazador en condiciones de normalidad tiene lugar en los cristales de hidroxiapatita recién formados y en las fibras de colágeno inmaduras, dependiendo del aporte sanguíneo, de la difusión extravascular y 
de la actividad osteoblástica. Globalmente nos informa de la respuesta dinámica metabólica del tejido óseo reflejando procesos fisiológicos y patológicos. Como prueba diagnóstica posee gran sensibilidad y poca especificidad, ya que cualquier alteración antes que cambios estructurales, produce cambios metabólicos. Estos, conllevan un aumento del flujo sanguíneo a ese nivel, con el consiguiente aumento de la fijación del radiotrazador en el hueso en comparación con el hueso simétrico o con las zonas vecinas. Mucho menos frecuentes son las imágenes hipocaptantes o isocaptantes como reflejo de procesos patológicos.

\section{Radiotrazadores}

La historia de los radiotrazadores actuales se inicia con la introducción del $99 \mathrm{mTc}$-pertecnetato y su posterior reducción con cloruro de estaño (20) que hizo posible marcar compuestos de fosfato con $99 \mathrm{mTc}$, obteniéndose así un radiotrazador ideal como los compuestos de fosfato con gran afinidad por los cristales de hidroxiapatita de la fase mineral ósea, especialmente por los lugares de nueva formación, marcados con un isótopo de propiedades físicas casi ideales: a) corto $11 / 2$ físico de $6 \mathrm{~h}, \mathrm{~b}$ ) emisión gamma monoenergética fácilmente colimable, c) ausencia de emisión beta, d) producción barata y cómoda y e) posibilidad de existir en varios estados de valencia que permiten la preparación de diferentes tipos de trazadores. Tras las primeras GO con polifosfatos, las investigaciones se dirigieron hacia los pirofosfatos. Con ellos se consiguió mejorar la relación actividad ósea/actividad de fondo. Finalmente, los difosfonatos combinaban las características biológicas de los anteriores con la resistencia in vivo a la destrucción enzimática por las fosfatasas tisulares.

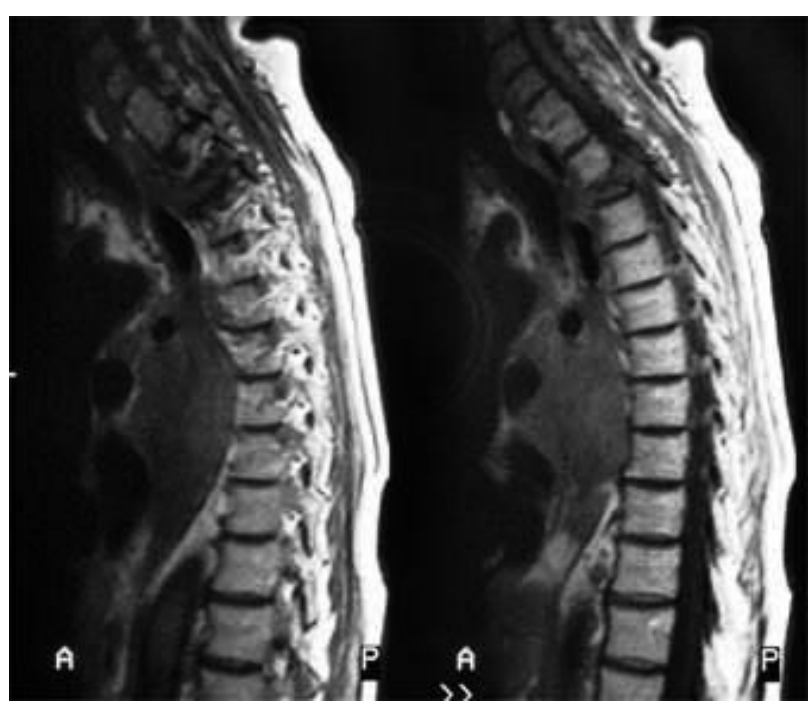

FIGURA 4. RM de columna en un paciente con metástasis ósea dorsal y compromiso medular.

\section{Técnica y adquisición de imágenes}

Tras la inyección endovenosa del radiotrazador, las imágenes se pueden obtener en tres momentos diferentes en el proceso de incorporación de éste al hueso. En una primera fase o fase de flujo sanguíneo, se obtienen imágenes dinámicas vasculares cada 2-3 segundos y durante 30 segundos -1 minuto a partir de la inyección endovenosa del radiotrazador. Con estas imágenes se aprecia la llegada de trazador y la perfusión de la zona a explorar, tanto hueso como tejidos blandos adyacentes. En una segunda fase, fase precoz o de pool sanguíneo, tras el primer minuto post-inyección, se obtienen imágenes estáticas o de cuerpo entero. El radiotrazador se encuentra entonces en el espacio vascular principalmente, pero ya empieza a salir del mismo y a incorporarse al hueso. Esta fase refleja las alteraciones en el fluido extracelular óseo que resultan de los cambios en la permeabilidad capilar. En una tercera fase, o fase ósea propiamente dicha, se obtienen imágenes estáticas estándares o de cuerpo entero. El momento de realización de estas imágenes varía. La captación ósea es máxima a las 2 horas, pero algunos autores creen que vale la pena esperar hasta las 4 horas para conseguir una mínima retención del radiotrazador en tejidos blandos y obtener así una máxima captación diferencial de las lesiones $(21,22)$. Como los difosfonatos tienen eliminación renal, entre la segunda y la tercera fase se recomienda al paciente que ingiera abundantes líquidos para forzar la diuresis, de modo que a las 2 horas se habrá fijado en el hueso el $50-60 \%$ de la dosis inyectada y el resto se va eliminando por orina. Del mismo modo, se disminuye la dosis absorbida por la vejiga. Justo antes de realizar la exploración, el paciente tiene que realizar una micción con el objeto de vaciar la vejiga nuevamente, eliminando asi el artefacto que produciría la gran actividad urinaria en las imágenes pélvicas.

Algunos autores proponen también la realización de imágenes más tardías, a las 24 horas $(23,24)$. En general, se recomienda realizar exploraciones que incluyan todo el cuerpo, aunque la zona a estudiar sea localizada. A menudo se han de realizar imágenes suplementarias para optimizar la visualización de ciertas estructuras. Esto se puede realizar de diferentes formas: a) variando la posición del paciente o la del cabezal de la gammacámara para obtener imágenes en cualquier posición (25), b) cambiando el colimador habitual por un pin-hole que magnifica las imágenes (26), o c) utilizando la técnica tomográfica SPECT (Single Photon Emission Computed Tomography), que permite una localización más precisa de las lesiones observadas en las imágenes planares - bien investiga zonas sospechosas sin alteraciones aparentes (27). 


\section{GO en el CP}

En la actualidad existe acuerdo sobre el papel de la $G O$ en el diagnóstico de la afectación metastásica ósea por CP. La valoración de las imágenes puede realizarse de forma cualitativa para la detección o cuantitativa como factor pronóstico. Mientras la sensibilidad de la GO es muy alta, también puede verse afectada por gran variedad de procesos benignos, así que la especificidad no es muy elevada (28). En las metástasis por CP la fase de formación de hueso estromal es más temprana y cuantitativamente más importante. Además de la osificación intramembranosa, se produce hueso nuevo reactivo como respuesta al estrés en el hueso debilitado y enfermo. Milch y Changus $(29,30)$ lo consideraron como un intento de reparar las alteraciones producidas por el cáncer. Este marcado aumento en la producción de hueso nuevo reactivo inmaduro tiene lugar en la gran mayoría de las metástasis, independientemente de la localización primaria del tumor. El hueso nuevo, tiene una gran avidez por los isótopos de captación ósea, lo que explica porqué la GO puede utilizarse para la detección precoz de metástasis esqueléticas $(29,31)$. Galasko et al. (32) han demostrado que los radiotrazadores óseos se acumulan mejor en las lesiones óseas focales recientes cuando el osteoide inmaduro está siendo formado o sólo está parcialmente calcificado. Cuando la mineralización del hueso nuevo reactivo es completa y estable, por ejemplo tras tratamiento hormonal efectivo, las GO pueden ser normales, incluso con lesiones osteoblásticas extensas apreciadas por radiografías. Por el contrario durante la terapia hormonal la intensidad de captación de los radiotrazadores en algunas m.o. puede incrementarse transitoriamente, fenómeno conocido como flare.

\section{Características de la imagen de las m.o.}

La imagen característica de una metástasis en la GO es un aumento focal de captación del radiotrazador, producida por respuesta osteoblástica que tiene lugar por la invasión ósea. Con frecuencia las metástasis son múltiples y se observan áreas irregularmente distribuidas de tamaño, forma e intensidad variable, (Figura 5). En la afectación muy extensa, la imagen puede ser muy parecida a la producida por las enfermedades metabólicas óseas. En estos casos recibe nombre de superscan y se caracteriza por un aumento generalizado de la captación del radiotrazador por el esqueleto axial, ausencia de visualización de calota craneal, poca actividad de fondo y escasa o nula visualización las imágenes renales. A menudo la hipercaptación esquelética es ligeramente irregular, en especial en costillas (33). Cuando las metástasis no producen respuesta osteoblástica, la GO puede ser normal. También puede existir una gammagrafía normal cuando la afectación metastásica todavía está confinada a la médula ósea $(34,35)$.
Otra posibilidad es la aparición de zonas con disminución de la captación del radiotrazador o lesiones fotopénicas. Kober et al. (36) comunicaron que hasta un $2 \%$ de las lesiones metastásicas producían este tipo de imágenes. En ocasiones, este tipo lesión está rodeado por un halo de aumento de captación del radiotrazador

Además del aspecto, el número de zonas con captación patológica del trazador refuerza la impresión diagnóstica. Será más difícil el diagnóstico cuando sólo existe una única área con aumento de captación. Alrededor de un $7 \%$ de los pacientes con metástasis presentarán una lesión única en la GO, mientras que si tomamos el conjunto de las lesiones únicas, hasta un $55 \%$ serán metastásicas (37-39). Es importante tener en cuenta que cuando la lesión es única, en algo más del $40 \%$ estará situada en el esqueleto axial, pero en más del $15 \%$ su localización será apendicular. Cuando la afectación metastásica es múltiple, el esqueleto axial está afectado en más del $60 \%$ y el apendicular en más del $15 \%$.

También puede ayudar la localización de las lesiones. El esqueleto axial está más frecuentemente afectado que el apendicular, correlacionándose bien con la distribución de médula ósea roja persistente en adultos. Hasta el $90 \%$ de las metástasis se desarrollan en la médula ósea antes de extenderse al hueso compacto. Además, la columna, pelvis y costillas son los lugares donde primero se localizan las metástasis y el cráneo, fémur, húmero, escápula y esternón se afectan en general en estadios más tardíos (40).

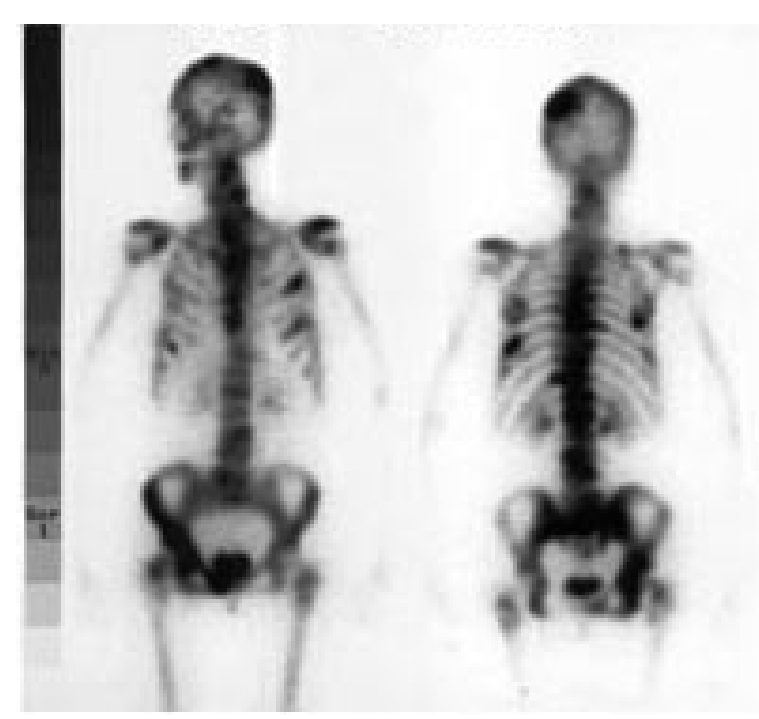

FIGURA 5. Gammagrafía ósea con imágenes hipercaptantes compatibles con metástasis óseas múltiples. 
Por último, la forma de la lesión puede ser muy orientativa. Así, una lesión elongada en una costilla tendrá más posibilidades de ser metastásica, mientras que una lesión puntual es más posible que tenga origen traumático.

\section{Estratificación}

A pesar de existir largas remisiones e incluso resolución excepcional de algún caso (41), en general la presencia de enfermedad metastásica ensombrece el pronóstico. Sin embargo, los intentos de cuantificación de la enfermedad ósea para convertirla en mesurable van dirigidos precisamente a establecer subgrupos de importancia pronostica, puesto que tanto la extensión como la localización de las metástasis óseas parecen afectar a la supervivencia. Ha sido probado que, aún en presencia de m.o., los pacientes con afectación axial pura o mínima afectación apendicular, afectación confinada a la pelvis y lesión única o menos de 6 lesiones hipercaptantes, tienen mejor pronóstico. No obstante, la cuantificación de la diseminación ósea presenta evidentes dificultades para valorar el volumen de la afectación y todas las mediciones son de tipo semicuantitativo. Soloway et al. (42) introdujeron en 1988 en la práctica clínica una estratificación de las m.o. en función de la extensión objetivada en la $\mathrm{GO}$ en el momento del diagnóstico inicial (EOD, Extensión Of Disease). Para la cuantificación se toma como unidad hipercaptante el equivalente a la mitad de un cuerpo vertebral obteniéndose así cinco grupos de afectación: a) 0 , normal o anormal pero debido a proceso benigno; $b$ ) 1, menos de seis lesiones hipercaptantes; c) 2, entre seis y veinte lesiones; d) 3 , más de veinte lesiones y e) 4 , imagen superscan o sus equivalentes -por ejemplo, más del $75 \%$ de afectación de costillas, vértebras y huesos pélvicos-, (Figura 6). Posteriormente, Yamashita et al. (43) objetivaron que la distribución de las metástasis en relación a su presencia axial o apendicular es un factor pronóstico importante y que no se correlacionaba con el EOD.

\section{GO por recidiva bioquímica tras terapia con intención curativa.}

La recidiva bioquímica se suele observar mucho antes de que se pueda detectar una positivización de la GO. Cher et al. (44) demostraron la utilidad de la GO para la evaluación de la recidiva bioquímica después de cirugía radical en 93 pacientes. Tras un análisis multivariante, el nivel de PSA y la velocidad de PSA eran las únicas variables predictivas de la positividad de la GO. La probabilidad de una $\mathrm{GO}$ positiva era inferior al $5 \%$ con el PSA por debajo de $40 \mathrm{ng} / \mathrm{ml}$, (Figura 7), mientras que la velocidad de PSA más sugestiva de m.o. era superior a 5 $\mathrm{ng} / \mathrm{ml} / \mathrm{mes}$. Se concluyó que la $\mathrm{GO}$ desempeñan un papel limitado en la evaluación de los pacientes con recidiva precoz tras terapia con intención curativa ya que los valores o incrementos bajos de PSA están asociados a exploraciones negativas.

\section{Tomografía por emisión de positrones (PET)}

Existe poca experiencia acumulada acerca de la utilidad de la PET para el diagnóstico de las m.o. en CP. Los estudios realizados hasta la fecha arrojan resultados poco esperanzadores para esta aplicación. Para Effert et al. (45) la PET con 18F-FDG no permitió detectar el $\mathrm{CP}$ metastásico, mientras que para Shreve et al. (46) sobre 202 focos de m.o., la sensibilidad de la PET fue de 65\% (131/202) con un valor predictivo positivo de $98 \%(131 / 133)$. Estos resultados son debidos a la baja tasa metabólica del CP y no superan los de las técnicas ya existentes. Los resultados con 11C-Colina y $11 \mathrm{C}$-Acetato supera los resultados del 18F-FDG con una sensibilidad, especificidad, VPP, VPN y eficacia de 100\%, 96\%, 92\%, $87 \%$ y $88 \%$ respectivamente $(47)$.

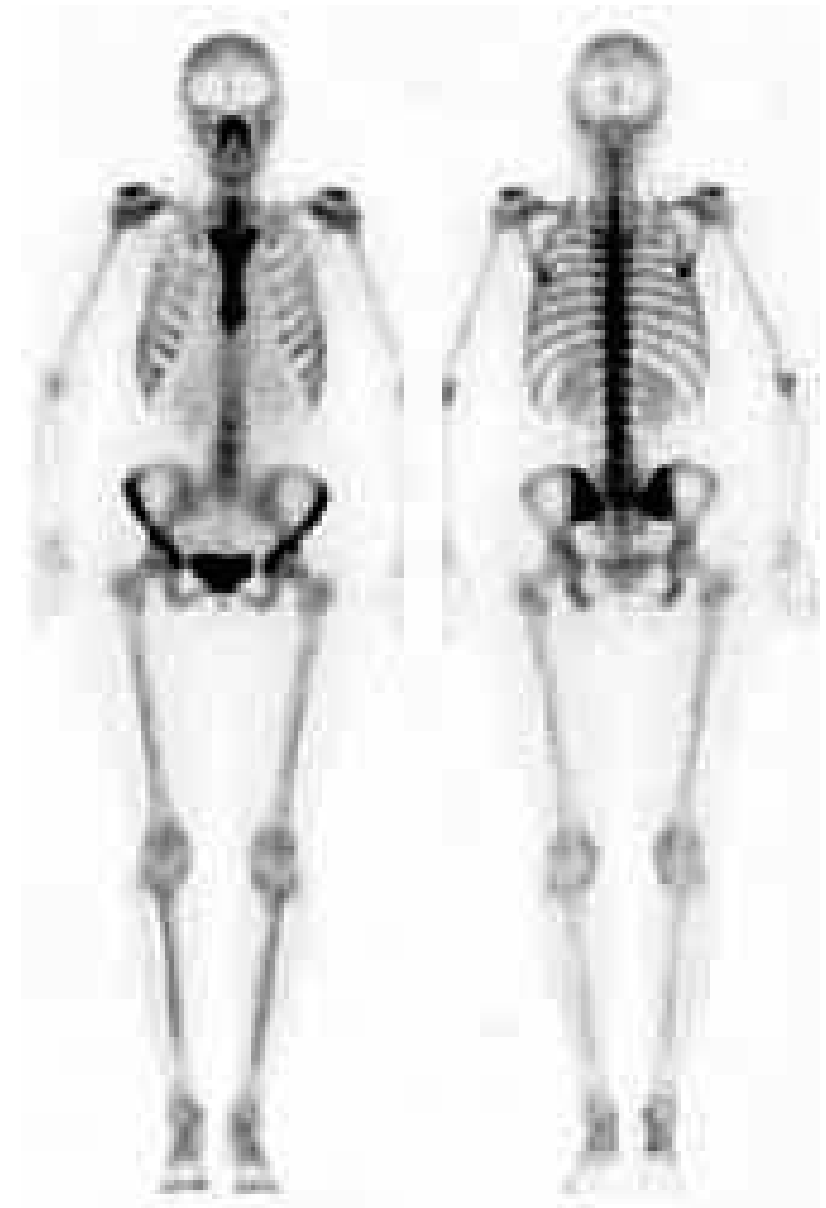

FIGURA 6. Gammagrafía ósea de un paciente con cáncer de próstata y PSA de 2000 ng/mL con patrón de superscan. 


\section{Gammagrafía e inmunogammagrafía medular}

La gammagrafía e inmunogammagrafía medular son técnicas no rutinarias en el diagnóstico de las m.o. por CP. Pueden ser empleadas en situaciones concretas en diagnóstico dudoso. Ito et al. (48) sugieren que la gammagrafía medular con coloides puede contribuir al diagnóstico de una manera más precoz que la GO, sin embargo, la gran captación hepática y esplénica dificultan la visualización y la técnica no tiene aceptación general. La inyección de nanocoloides marcados con $99 \mathrm{~m}$-Tc viene seguida de su captación por parte de las células del sistema reticuloendotelial y de la visualización principalmente de áreas medulares, hepáticas y esplénicas. El desplazamiento de esta población por células metastásicas es la base para la visualización de áreas frías en la gammagrafía medular. Para Haddock et al. (49) , esta técnica es menos sensible que la GO pero puede contribuir a aclarar diagnósticos dudosos.

\section{Aportación del laboratorio para el estudio de las m.o.}

Durante la última década, la mayoría de los estudios clínicos sobre la diseminación del $\mathrm{CP}$ se han centrado en la capacidad del PSA para predecir el resultado de la GO. No parece existir un consenso sobre qué concentración de PSA predice la existencia de m.o. Para Miller et al. (50) en una población con una prevalencia de enfermedad metastásica del $23 \%$, el $71 \%$ de los pacientes con m.o. presentaron PSA mayor de $20 \mathrm{ng} / \mathrm{ml}$ en el momento del diagnóstico obteniendo un valor predictivo positivo (VPP) de $38 \%$ y un valor predictivo negativo (VPN) de $88 \%$. Rana et al. (51) encuentran para un PSA de $100 \mathrm{ng} /$

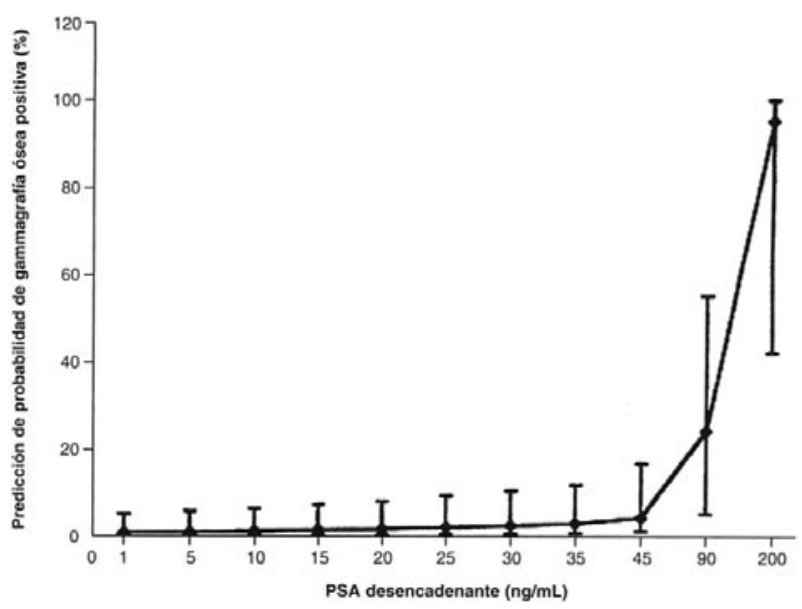

FIGURA 7. Predicción de la probabilidad con 95\% de intervalo de confianza de resultado positivo de la gammagrafía ósea en el seguimiento de los pacientes con recidiva bioquímica tras prostatectomía radical (tomado de Cher et al.) $\mathrm{ml}$ un VPP de 100\%, indicando correctamente el $83 \%$ de los pacientes con m.o. Resultados similares han sido confirmados por otros grupos. Para Pantelides et al. (52) un PSA superior a $58 \mathrm{ng} / \mathrm{ml}$ es altamente indicativo de diseminación ósea mientras que para Gerber et al. (53) un PSA de $25 \mathrm{ng} / \mathrm{ml}$ presenta un VPP de $41 \%$. De todo esto se deduce que no existe un consenso a la hora de determinar que concentración de PSA inicial predice la existencia de m.o.

Mayor interés clínico han tenido los estudios dirigidos a determinar que concentración de PSA permite estimar que un paciente no presenta con seguridad m.o. En este sentido, el trabajo de Chybowski et al. (54) es un estudio de referencia. Estos autores concluyeron que la concentración de PSA muestra una correlación significativa con la incidencia de gammagrafía positiva, que es el mejor parámetro predictivo de los hallazgos gammagráficos y que una concentración de PSA de $20 \mathrm{ng} / \mathrm{ml}$ presenta un VPN de $99.7 \%$. Se sugirió que no era necesaria la realización de estudios dirigidos al diagnóstico de las m.o. en los pacientes con PSA inicial por debajo de ese valor. En esta misma línea, Oesterling et al. (55) analizaron las implicaciones económicas de esta recomendación y propusieron que no se realizara la gammagrafía a los pacientes con PSA inferior a 10 $\mathrm{ng} / \mathrm{ml}$ en ausencia de sintomatología, dada la alta probabilidad de que resulte negativa. Los mismos autores reconocieron que estos resultados podrían proceder de una población con sesgo de selección. Otra potencial crítica se dirigió en la línea de que aunque poco probable era posible practicar un tratamiento inadecuado a un paciente con m.o., demostrado que existen CP que expresan poco PSA. En las poblaciones con una prevalencia de enfermedad metastásica mayor $y$ en el controvertido rango de PSA entre 10 y $20 \mathrm{ng} / \mathrm{ml}$ se ha propuesto el empleo combinado de la concentración sérica de PSA y la de los marcadores del metabolismo osteoblástico. De esta manera se pueden llegar a evitar hasta un tercio de las exploraciones gammagráficas diagnosticando correctamente a todos los pacientes (56).

\section{CONCLUSIÓN}

En el estudio de la diseminación a distancia en el CP se han producido avances en los últimos años dirigidos a conseguir una valoración más eficiente del estadio clínico. Al perfeccionamiento de las técnicas de imagen se ha asociado la evolución en la indicación de las exploraciones complementarias siguiendo criterios bioquímicos y la aparición de las nuevas técnicas de estudio de los procesos metabólicos en el tejido creando el diagnóstico por la imagen molecular. Algunas de estas técnicas se encuentran 
actualmente en evaluación y pueden contribuir en un próximo futuro a cambiar la práctica clínica del estudio de extensión. En el estudio de la diseminación ganglionar, el TC o la RM son las técnicas por imagen estándar indicadas cuando el PSA es superior a $20 \mathrm{ng} / \mathrm{ml}$, en un estadio local $\geq \mathrm{T} 3$ o en un CP de alto grado. En el estudio de la diseminación ósea, la GO continúa siendo el rastreo de rutina, indicándose en caso de PSA superior a $20 \mathrm{ng} / \mathrm{ml}, \mathrm{CP}$ de alto grado o sintomatología ósea.

\section{BIBLIOGRAFIA y LECTURAS RECOMENDADAS ( ${ }^{*}$ lectura de interés $y^{* *}$ lectura fundamental)}

1. HUNCHAREK, M.; MUSCAT, J.: "Serum prostate specific antigen as a predictor of the staging abdominal/pelvic computed tomography in newly diagnosed prostate cancer". Abdom. Imaging., 21: 364, 1996.

2. YU, K.K.; HRICAK, H.: "Neoplasms of the prostate gland. Clinical Urography". Second Edition. Saunders Ed. 2000.

3. OYEN, R.H.; VAN POPPEL, H.P.; AMEYE, F.E. y cols.: "Lymph node staging of localized prostate carcinoma with CT and CT-guided fine-needle aspiration biopsy: Prospective study of 285 patients". Radiology, 190: 315, 1994.

4. LANGLOTZ, C.; SCHNALL, M.; POLLACK, H.: "Staging of prostatic cancer: Accuracy of MR imaging". Radiology, 194: 645, 1995.

5. SELTZER, M.A.; BARBARIE, Z.; BELLDEGRUN, A. y cols.: "Comparison of helical computerized tomography, positron emisión tomography and monoclonal antibody scans for evaluation of lymph node metastases in patients with prostate specific antigen relapse alter treatment for localized prostate cancer". J. Urol., 162: 1322, 1999.

6. DE JONG, I.J.; PRIUM, J.; ELSINGA, P.H. y cols.: "Preoperative staging of pelvic lymph nodes in prostate cancer by 11C-Choline PET". J. Nucl. Med., 44: 331, 2003.

*7. LANGE, P.H.: "ProstaScint scan for staging prostate cancer". Urology, 57: 402, 2001.

8. EDELSTYN, G.A.; GILLESPIE, P.J.; GREBELL, F.S.: "The radiological demonstration of osseous metastases: experimental observation". Clin. Radiol., 18: 158, 1967.

9. BORAK, J.: "Relationship between the clinical and roentgenological findings in bone metastases”. Surg. Gynecol. Obst., 75: 599, 1942.

10. LOTE, K.; WALLOE, A.; BJERSAND, A.: "Bone metastasis. Prognosis, diagnosis and treatment". Acta Radiol. Oncol., 25: 227, 1986.

11. HOVSEPIAN, J.A.; BYAR, D.P.: "Quantitative radiology for staging and prognosis of patients with advanced prostatic carcinoma". Urology, 14: 145, 1979.

12. MOORE, S.G.; DAWSON, K.L.: "Red and yellow marrow in the femur: age related changes in appearance at MR imaging”. Radiology, 175: 219, 1990.

13. SIGIMURA, K.; YAMASAKI, K.; KITAGAKI, H.: "Bone marrow diseases of the spine: differentiation with $\mathrm{T} 1$ and $\mathrm{T} 2$ relaxation times in $\mathrm{MR}$ imaging". Radiology, 165: 541, 1987.

14. BAFER, L.L.; GOODMAN,S.B.; PERKESH, I. y cols.: "Benign versus pathologic compression fractures of vertebral bodies: assessment with conventional spin echo, chemical shift and STIR MR imaging". Radiology, 174: 495, 1990.

15. DAFFNER, R.H.; LUPETIN, A.R.; DASH, J. y cols.: "MRI in the detection of malignant infiltration of bone marrow". AJR, 146: 353, 1986.

16. MEHTA, R.C.; WILSON, M.A.; PERTMAN, S.B.: "False negative bone scan in extensive metastatic disease: CT and MRI findings". JCAT, 13: 717, 1989.

*17. AVRAHAMI, E.; TADMOR, R.; DALLY, O. y cols.: "Early demonstration of spinal metastases in patients with normal radiography and CT and radionuclide bone scan". JCAT, 13: 598, 1989.

*18. WETZEL, L.H.; ENGELBRECHT, D.E.; BAXTER, K.G. y cols.: "Comparison of MR imaging and bone scintigraphy for detection and evaluation of osseous spinal metastases". Radiology, 177: 371, 1990.

19. FUJI, Y.; HIGASHI, Y.; OWADA, F. y cols.: "Magnetic resonance imaging for the diagnosis of prostate cancer metastatic to bone". Br. J. Urol., 75: 54, 1995.

20. ECKELMAN, W.; RICHARDS, P.: "Instant 99mTc-DTPA". J. Nucl. Med., 11: 761, 1970.

21. CITRIN, D.L.; BESSENT, R.G.; McGINLEY, E. y cols.: "Dynamic studies with 99mTc-HEDP in normal subjects and in patients with bone tumours". J. Nucl. Med., 16: 886, 1975.

22. FOGELMAN, I.; CITRIN, D.L.; McKILLOP, J.H. y cols.: "A clinical comparison of 99mTc-HEDP and $99 \mathrm{mTc}-\mathrm{MDP}$ in the detection of bone metastases: concise communication". J. Nucl. Med., 20: 98, 1979.

23. MERRICK, M.V.: "Review article. Bone scanning”. Br. J. Radiol., 48: 327, 1975.

24. HARDOFF, R.; FRONT, D.: "The value of delayed bone scintigraphy". Clin. Nucl. Med., 3: 39, 1978.

25. SCHUTTLE, H.E.: "Some special views in bone scanning". Clin. Nucl. Med., 5: 172, 1980.

26. BAHK, Y.W.; KIM, O.K.; CHUNG, S.K. y cols.: "Pinhole collimator scintigraphy in differential diagnosis of metastasis, fracture and infections of the spine". J. Nucl. Med., 28: 447, 1987.

27. SHIH, W.J.; MAGOUN, S.; STRIPP, V. y cols.: "Volume three-dimensional display of $99 \mathrm{mTc}$ - 
HMDP skull, cervical vertebral, thoracic vertebral, lumbar vertebral, and/or pelvic SPECT images by triple head camera". J. Nucl. Med., 33: 913, 1991.

28. O'MARA, R.E.: "Skeletal scanning in neoplastic disease". Cancer, 37: 480, 1986.

29. GALASKO, C.S.B.: "Mechanisms of lytic and blastic metastatic disease of bone". Clin. Orthop. Rel. Res., 12: 20, 1982.

30. MILCH, R.A.; CHANGUS, G.W.: "Response of bone to tumour invasion". Cancer, 9: 340, 1956.

31. GALASKO, C.S.B.: "Mechanism of uptake of bone imaging isotopes by skeletal metastases". Clin. Nucl. Med., 5: 565, 1980.

32. GALASKO, C.S.B.: "The pathological basis for skeletal scintigraphy”. J. Bone Joint Surg., 57: 353, 1975.

33. FOGELMAN, I.; McKILLOP, J.H.; GREIG, W.R. y cols.: "Absent kidney sign associated with symmetrical and uniformly increased uptake of tracer by skeleton”. Eur. J. Nucl. Med., 2: 257, 1977.

34. DUNCKER, C.M.; CARRIÓ, I.; BERNÁ, L.L.: "Radioimmune imaging of bone marrow in patients with suspected bone metastases from primary breast cancer". J. Nucl. Med., 31: 1450, 1991.

35. SANAL, S.M.; FLICKINGER, F.W.; CAUDELL, M.J. y cols.: "Detection of bone marrow involvement in breast cancer with magnetic resonance imaging”. J. Clin. Oncol., 12: 1415, 1994.

36. KOBER, B.; HERMANN, H.J.; WETZEL, E.: "Cold lesions in bone scintigraphy". Fortschr. Roentgenstr., 131: 545, 1979.

37. CORCORAN, R.J.; THRALL, J.H.; KYLE, R.W.: "Solitary abnormalities in bone scan of patients with extraosseous malignancies". Radiology, 121: 663, 1976.

38. RAPPAPORT, A.H.; HOFFER, P.B.; GENANT, H.K. y cols.: "Unifocal bone findings by scintigraphy. Clinical significance in patients with know primary cancer". West J. Med., 129: 188, 1978.

39. BROWN, M.L.: "Significance of the solitary lesion in paediatric bone scanning". J. Nucl. Med., 24: 114, 1987.

40. YAXAWA, Y.; FRASSICA, F.J.; CHAO, E.Y.S.: "Metastatic bone disease: a study of the surgical treatment of 166 pathologic humeral and femoral fractures". Clin. Orthop., 25: 213, 1980.

41. SCHURMANS, J.R.; BLIJENBERG, B.G.; MICKISH, G.H. y cols.: "Spontaneous remission of a body metastasis in prostatic adenocarcinoma". J. Urol., 155: 653, 1996.

**42. SOLOWAY, M.S.; HARDEMAN, S.W.; HICKEY, D. y cols.: "Stratfication of patients with prostate cancer based on extent of disease on initial bone scan". Cancer, 61: 195, 1988.

**43. YAMASHITA, K.; DENNO, K.; UEDA, T. y cols.: "Prognostic significance of bone metastases in patients with metastatic prostate cancer". Cancer, 71: 1297, 1993.

*44. CHER, M.L.; BIANCO, F.J.; LAM, J.S. y cols.: "Limited role of radionuclide bone scintigraphy patients with prostate specific antigen elevations after radical prostatectomy". J. Urol., 160: 1387, 1998.

**45. EFFERT, P.J.; BARES, R.; HANDT, S. y cols.: "Metabolic imaging of untreated prostate cancer by positron emission tomography with 18fluorinelabeled deoxyglucose". J. Urol., 155: 994, 1996.

46. SHREVE, P.D.; GOSSMAN, H.B.; GROSS, M.D. y cols.: "Metastatic prostate cancer: initial findings of PET with 2-deoxy-2-F18-fluoro-Dglucose". Radiology, 199: 751, 1996.

**47. DE JONG, I.J.; PRIUM, J.; ELSINGA, P.H. y cols.: "Visualization of prostate cancer with 11CCholine positron emission tomography". Eur. Urol., 42: 18, 2002.

48. ITO, Y.; OKUYAMA, S.; SUZUKI, M.: “Bone marrow scintigraphy in the early diagnosis of experimental metastatic carcinoma". Cancer, 31: 1222, 1973.

49. HADDOCK, G.; GRAY, H.W.; McKILLOP, J.H. y cols.: "99mTc-Nanocolloid bone marrow sintigraphy in prostate cancer". Br. J. Urol., 63: 497, 1989.

*50. GLEAVE, M.E.; COUPLAND, D.; DRACHENBERG, D. y cols.: "Ability of serum prostate specific antigen levels to predict normal bone scan in patients with newly diagnosed prostate cancer". Urology, 47: 708, 1996.

51. PUPPO, P.; PERACHINO, M.: "New markers of bone metastasis from prostate cancer: a significant saving”. European Urology Today, Personal Communications, 8, 1995.

52. MOROTE, J.; RUIBAL, A.; PASCUAL, C.: "Bone marrow prostatic specific antigen and prostatic acid phosphatase levels: are they helpful in staging prostate cancer?". J. Urol., 137: 891, 1987.

53. DEFTOS, L.J.: "Bone protein and peptide assays in the diagnosis and management of skeletal disease". Clin. Chem., 37: 1143, 1991.

**54. CHYBOWSKI, F.M.; KELLER, J.J.; BERGSTRALH, E.J. y cols.: "Predicting radionuclide bone scan findings in patients with newly diagnosed untreated prostate cancer: prostate specific antigen is superior to all other clinical parameters". J. Urol., 145: 313, 1991.

**55. OESTERLING, J.E.; MARTIN, S.K.; BERGSTRALH, E.J. y cols.: "The use of prostate specific antigen in staging patients with newly diagnosed prostate cancer". JAMA, 269: 57, 1993.

*56. LORENTE, J.A.; VALENZUELA, H.; MOROTE, J. y cols.: "Serum bone alkaline phosphatase levels enhance the clinical utility of prostate specific antigen in the staging of newly diagnosed prostate cancer patients". Eur. J. Nucl. Med., 26: $625,1999$. 